\title{
Tissue Engineering and Regenerative Medicine 2019: The Role of Biofabrication-A Year in Review
}

\author{
Tiago Ramos, $\mathrm{PhD}^{1}$ and Lorenzo Moroni, $\mathrm{PhD}^{2}$
}

\begin{abstract}
Despite its relative youth, biofabrication is unceasingly expanding by assimilating the contributions from various disciplinary areas and their technological advances. Those developments have spawned the range of available options to produce structures with complex geometries while accurately manipulating and controlling cell behavior. As it evolves, biofabrication impacts other research fields, allowing the fabrication of tissue models of increased complexity that more closely resemble the dynamics of living tissue. The recent blooming and evolutions in biofabrication have opened new windows and perspectives that could aid the translational struggle in tissue engineering and regenerative medicine (TERM) applications. Based on similar methodologies applied in past years' reviews, we identified the most high-impact publications and reviewed the major concepts, findings, and research outcomes in the context of advancement beyond the state-of-the-art in the field. We first aim to clarify the confusion in terminology and concepts in biofabrication to therefore introduce the striking evolutions in three-dimensional and four-dimensional bioprinting of tissues. We conclude with a short discussion on the future outlooks for innovation that biofabrication could bring to TERM research.
\end{abstract}

Keywords: biofabrication, bioprinting, bioassembly, instructive scaffolds

\section{The Aim and Scope}

$\mathbf{T}$ HE FIELD OF TISSUE engineering and regenerative medicine (TERM) is growing by combining different disciplinary approaches, including stem cell biology, functional scaffold materials, nanotechnology, and the most recent additive manufacturing (AM) (commonly known as threedimensional $[3 \mathrm{D}]$ printing). AM methods have also made great and rapid advancements and are now utilized to produce structures with complex geometries used in a wide variety of fields. More importantly for the scope of this review, AM has made important progress in the production of a series of constructs designed and used for medical engineering approaches. AM technology has also been at the foundation of biofabrication, a quickly growing interdisciplinary field that has recently been the focus of great attention with significant updates on its terminology, particularly with the adoption of biofabrication concepts within the TERM fields. In view of the potential of biofabrication strategies to impact TERM applications with the generation of constructs of enhanced functionality, this article also aims to focus on the most relevant topics related to biofabrication over the last year (or so).

Some excellent reviews ${ }^{1-5}$ and other "year in review" articles $^{6-8}$ have been recently published regarding the AM and biofabrication concepts applied in TERM. Here, we have adopted similar methods to those used in previous "year in review" articles to select the main topics to be highlighted. First, we searched on the National Center for Biotechnology Information (NCBI) database for the keywords "biofabrication," "additive manufacturing," "bioprinting," or "bioassembly" and sorted publications from January 1, 2018 to November 30, 2019. Second, we have examined previous "year in review" article topics to carefully exclude those already discussed. Third, in this review, we have excluded the extensively reviewed field of cell/ biomaterial interaction, as this is of broader scope than biofabrication. Fourth and finally, we have selected three main topics to cover in this review: (1) efforts in clarifying terminology and (2) 3D and (3) four-dimensional (4D) bioprinting of tissues. For each section, several articles are used to illustrate the major concepts, findings, and research outcomes in the context of advancement beyond the stateof-the-art in the field.

\section{Clarifying Terminology-Biofabrication and Bioinks}

Over the last 3 years the "biofabrication community" has made an important effort to clarify the terminology related to "biofabrication"1-3 and "bioinks," applications. First coined in 1994, "biofabrication" and its

\footnotetext{
${ }^{1}$ Institute of Ophthalmology, University College of London, London, United Kingdom.

${ }^{2}$ Complex Tissue Regeneration Department, MERLN Institute for Technology-Inspired Regenerative Medicine, Maastricht University, Maastricht, the Netherlands.
} 
related terminology have evolved over time in parallel with the concept of bioprinting. This parallelism has led to confusion in terminology and concepts and in some instances to a conflation of the two terms. Recent reviews have extensively addressed this matter and reappraised the definition of these terms. ${ }^{1,3}$ Biofabrication has been defined by several groups over the years. ${ }^{9-12}$ Taking into account the evolving concepts and the wide panoply of research activities encompassed in the field of biofabrication, Groll et al. suggested in 2016 that biofabrication for TERM is "the automated generation of biologically functional products with structural organization from living cells, bioactive molecules, biomaterials, cell aggregates such as micro-tissues, or hybrid cell-material constructs, through Bioprinting or Bioassembly and subsequent tissue maturation processes.", 1 Within biofabrication, therefore, there are two main pillars distinguished by the length scale of the minimum fabrication unit: (1) "bioprinting" allows the spatial arrangement of cells, materials, and biological active factors, in which the minimum fabrication unit is down to molecular level; (2) "bioassembly" facilitates the automated assembly of cell containing building blocks, where the minimum fabrication units are preformed cell-containing building blocks with sizes large enough so that automated assembly can technologically be achieved. ${ }^{1}$ In addition, the fabrication of 3D scaffolds by AM technologies with hierarchical and/or surface properties able to steer cell activity is to be considered within biofabrication approaches for TERM applications when the subsequent maturation process yields a structural biologically functional construct. Thorough reviews on the technology and terminology used in biofabrication have recently been published elsewhere. ${ }^{1-3}$ These techniques can provide constructs with increasingly high levels of biomimicry toward getting closer and closer to the complexity of tissues and organs. More importantly, biofabrication allows for the spatiotemporal modulation of cell/ cell and cell/extracellular matrix (ECM) interaction. The most commonly used technologies applied in biofabrication comprise the following: (1) 3D and 4D printing; (2) lightbased technologies, including stereolithography (SLA), twophoton polymerization (2-PP), and laser-induced hydrogel degradation; (3) fused deposition modeling (FDM)/3D fiber deposition/bioextrusion; (4) 3D plotting/bioplotting/robotic dispensing/extrusion bioprinting; (5) wet-spun automated extrusion systems; (6) ink-jet and valve-jet bioprinting; and (7) electrospinning. Here, we aim to focus on the most recent advancements on these technologies and thereafter address the spatiotemporal resolutions that led to the rise of 4D bioprinting constructs.

First used in 2003, the term bioink was used in the context of bioprinting and was introduced together with the term biopaper. ${ }^{13,14}$ The initial concept was to provide a biopaper (hydrogel) that would be thereafter populated with living cells and/or tissue spheroids as the "bioink" to be bioprinted. Therefore, the term bioink originally referred to the cellular component of the solution that would be positioned in the $3 \mathrm{D}$ in a porous biomaterial (i.e., a scaffold) or within the hydrogel. In 2018, Williams et al. proposed a more complicated division of the term bioink in four subcategories: support bioinks, fugitive bioinks, structural bioinks, and functional bioinks. ${ }^{15}$ In the most recent studies, and according to the recently updated definition of biofab- rication, ${ }^{1}$ bioinks are described as "a formulation of cells suitable for processing by an automated biofabrication technology that may also contain biologically active components and biomaterials." 4 Therefore, cells are the mandatory component of a bioink, and thus, formulations that include biologically active components or molecules, but do not contain any cells, are not qualified as a bioink. Further distinction between bioinks and biomaterial inks was proposed recently by Groll et al. ${ }^{4}$ Biomaterial inks were therefore defined as "materials that can be printed and subsequently seeded with cells after printing, but not directly formulated with cells.',

\section{D Bioprinting}

Bioprinting is a class of AM processes in which biomaterials, bioactive molecules, and cells are combined and assembled to manufacture complex engineered structures for biological studies with particular interest for regenerative medicine. ${ }^{2,3}$ To date, there are four main strategies for bioprinting: extrusion-based (also referred to as bioplotting), drop-based (also referred to as inkjet or drop-on-demand), laser-based bioprinting (also known as laser-induced forward transfer [LIFT]), and SLA. ${ }^{16,17}$ Each of these techniques shows different ranges of resolution, manufacturing times, and limitations (extensively reviewed elsewhere ${ }^{3,17}$ ).

\section{Biomaterials Used in Bioprinting}

Biomaterials can be categorized regarding their source, from either natural or synthetic sources, and are grouped into static (nondegradable) and dynamic (biodegradable, through hydrolysis or enzymatic degradation). Materials used in biofabrication must meet specific criteria, depending on the fabrication technique (comprehensively reviewed in Moroni et $a .^{2}$ ). Historically, materials used in bioprinting are those that quickly stabilize from nonviscous states ${ }^{18,19}$ and can mainly be divided into hydrogels (reviewed in Gungor-Ozkerim et al., Bishop et al. ${ }^{20,21}$ ) and microcarriers (reviewed in Ashammakhi et al. ${ }^{22}$ ). To be suitable for extrusion bioprinting (the most commonly used biofabrication technique), a material must show steady flow until deposition and must rapidly stabilize upon delivery.

\section{Scaffold-Free Approach in Bioprinting}

In addition to scaffold-based bioprinting approaches, the so-called scaffold-free bioprinting technique has also emerged in the last decade. This consists in the use of cells or cell aggregates, without any support material for the bioprinting process. Therefore, providing a natural environment for cells promotes better cell/cell interaction, compared with the traditional 3D printing techniques. This technique may be of particular interest for induced pluripotent stem cells (iPSCs), until the discovery of new hydrogels that can better maintain iPSC viability and differentiation state.

\section{D Bioprinting Strategies-Nozzle-Based Bioprinting Techniques}

In vitro $3 \mathrm{D}$ models are of great advantage to investigate the safety and efficacy of several therapeutic agents for drug development and screening and to model dynamic biological processes. Numerous normal and diseased 3D tissue 
models have been developed and extensively reviewed elsewhere. Therefore, here we aim to explore the most recent findings in the last year on bioprinted in vitro tissue models and their potential applications.

\section{Vascular Tissues}

An extensive review stating materials, cells, and techniques used in bioprinting approaches with particular focus on vascular structures has been published elsewhere. ${ }^{17}$ In a breakthrough study, Attalla et al. presented a new microfluidic nozzle design capable of multiaxial extrusion to 3D print and pattern bi- and trilayered hollow channel structures. These nozzles allow for the structures to be embedded within layers of gels and ECM proteins in a quick and simple manner. Their design allows for multiaxial extrusion and wide versatility in material incorporation to create heterogeneous structures. ${ }^{23}$ These highly complex heterogeneous and hierarchical architectures show great potential applications on artificially fabricated tubular or fiber-like structures, including vascular and nervous tissue applications. In a recent study, xanthan gum was deposited into a $\mathrm{CaCl}_{2}$-infused gelatin to form sacrificial channels to rapid prototype microfluidic platforms with earlobe-shaped channels. ${ }^{24}$ Using this method, the authors created complex 3D structures, stable enough to support themselves as well as complex channel geometries that were evenly perfusable. ${ }^{24}$

To further develop potential new vascular tissues, Ji et al. deposited a sacrificial ink between several layers of a cell-laden oriented hydrogel bioink to therefore produce perfusable channels. Briefly, they constructed methacrylate alginate or methacrylate hyaluronic acid hydrogels with incorporated sacrificial ink (Pluronic), which was removed after curing. Mesenchymal stromal cells (MSCs) were included in the bioprinted matrix hydrogel and showed high viability, and endothelial cells (ECs) were perfused inside the channel, attaching inside the channels circumferentially to form confluent layers. The novelty of this method arises from the fact that the sacrificial ink and the matrix hydrogel were not mixed during the deposition process, which allowed for high resolution of the final channel after perfusion. ${ }^{25}$ With this approach, the authors have produced tunable channels with a large range of sizes (300 to $1500 \mu \mathrm{m}$ in diameter) and complexities, including a matrix with converged channels, without complex device modifications for bioprinters nor complicated methods for ink synthesis and processing as previously reported. ${ }^{26}$

Schöneberg et al. used drop-based bioprinting to generate in vitro blood vessel models. They separately deposited droplets of gelatin with ECs to form the core of the structure, followed by a layer of fibrinogen with smooth muscle cells, and an external layer of thrombin. This approach allowed for the crosslinking of the fibrinogen and the external thrombin to form an outer fibrin layer to surround the core material. Once completed the process, a collagen/fibrinogen based bioink containing fibroblasts was quickly crosslinked with thrombin. By doing so, the authors successfully reproduced the three layers of a blood vessel by reconstituting the tunica intima (endothelium), tunica media (elastic smooth muscle cells), and the tunica adventitia (matrix of fibroblasts). ${ }^{27}$ Additional work is still needed to engineer de novo vascular tissues, although the novel technique developed by
Schöneberg et al. is a valuable step forward in that direction as they achieved to manufacture perfusable vessel models with a biofunctional multilayer wall composition.

\section{Neural Tissue}

The complex physiology and the limited regenerative capacity of the neural tissue make the repair of this system extremely challenging. However, recent studies have utilized bioprinting techniques to seek for viable approaches. Extensive reviews on engineering 3D environments to increase neural cell viability ${ }^{28}$ and to recapitulate in vitro disease models of the central nervous system ${ }^{29}$ have recently been published. A promising study for disease modeling and spinal cord nervous tissue regeneration has been published by Joung et al. They first generated oligodendrocyte progenitor cells (OPCs) and spinal neuronal progenitor cells (SNPCs) from iPSCs. These two cell types were separately incorporated as clusters of either OPCs or SNPCs into precise positions within a biocompatible scaffold (made either of acetoxy-based vulcanizing silicone, poly(ethylene glycol) diacrylate, or hydrogels of alginate mixed with methylcellulose at different ratios). Successful bioprinting of OPC clusters in combination with SNPCs resulted in outgrowth of axons and associated OPCs, suggesting that SNPCs could successfully differentiate into mature neurons, and OPCs into oligodendrocytes to myelinate the axons. ${ }^{30}$ These constructs showed to be a suitable multicellular neural tissue engineering approach to model the complex central nervous system tissue architectures in vitro.

Ning et al. have used composite hydrogels of alginate, fibrin, hyaluronic acid, and arginylglycylaspartic acid (RGD) peptides encapsulated with Schwann cells to construct scaffolds with potential to repair nerves after injury. ${ }^{31}$ They have shown that Schwann cells were viable, proliferated, and aligned inside the hydrogels, therefore providing adequate cues to direct the extension of dorsal root ganglion neurites along the bioprinted strands.

\section{Skin}

Several approaches have been developed in the last year to manufacture skin-like substitutes as grafts to quickly reestablished its structure and function upon injury. Miguel et al. have combined electrospinning and bioprinting techniques to manufacture $3 \mathrm{D}$ skin construct mimics. A poly(caprolactone) and silk sericin blend was electrospun to produce a mimic of the epidermis, while the dermis layer was formed by depositing layer-by-layer a chitosan/alginate hydrogel. The results revealed constructs that displayed morphology, porosity, mechanical properties, wettability, and antimicrobial activity that grants their application as potential skin substitutes. In addition, these structures were incubated with fibroblasts (normal human dermal fibroblast [NHDF] cell line) and showed to support cell adhesion, migration, and proliferation. ${ }^{32}$ Together, the results showed that the top electrospun layer can act as protective barrier against microorganisms' infiltration, while the porous hydrogel constitutes a suitable structure for cell migration and proliferation.

Using a composite hydrogel of alginate and gelatin, Liu et al. have also developed bioprinted structures with promising potentials for future skin tissue engineering. ${ }^{33}$ 
Human epithelial cells and MSCs were bioprinted to form bilayered skin-like constructs of epidermis and the stromal layers in vitro, respectively. These constructs showed that rheological properties commensurate with their potential applications, including shear-thinning behavior and temperature-dependent viscosities, and support high cell viability. Similar studies were later performed by Cheng et al., ${ }^{34}$ however, alginate/gelatin-based bioinks were used to induce the differentiation of epidermal progenitors toward sweat gland-like cells They showed that MSCs injected on the alginate/gelatin-based bioink differentiate sweat glandlike cells expressing their keratin-associated markers after 7 days. In addition, the stiffness of the bioink showed to be similar to that of the mouse dermal tissue.

Derr et al. have manufactured a fully 3D bioprinted skin equivalent using a three-syringe configuration to extrude three independent solutions. To mimic the dermis, they prepared a hydrogel containing gelatin, fibrinogen, collagen type I, and elastin incorporated with fibroblasts, the basal layer mimic was composed of an acellular solution of laminin/ entactin, and the epidermis composed of solely keratocytes in the culture media. This bioprinted skin equivalent showed good barrier function, high cell viability, and morphologically, features similar to those of normal human skin, as given in Derr et al. ${ }^{35}$

Atala's group developed a novel approach, coined "BioMask." This is a customized engineered skin substitute combined with a wound dressing layer that fits onto the facial wound. Using computed tomography images of skin wounds in mice, they precisely dispensed dressing material and cell-laden hydrogels into the wound in a layerby-layer manner (Fig. 1A). The manufactured BioMask consisted of three layers: (1) a porous polyurethane layer, (2) a keratinocyte-laden hydrogel (hyaluronic acid, glycerol, gelatin, and fibrinogen) layer, and (3) a fibroblast-laden hydrogel (hyaluronic acid, glycerol, gelatin, and fibrinogen) layer. The 3D BioMask was then placed on a wound site previously implanted with a cartilage-like structure made of poly ( $\varepsilon$-caprolactone) on athymic mice models (Fig. 1B). The in vivo results showed skin contraction, and the histological examination revealed the regeneration of skin tissue consisting of epidermis and dermis layers. ${ }^{36}$ The same

A

Step 1 - Implantation of the cartilage construct on the mouse dorsal skin pocket

a

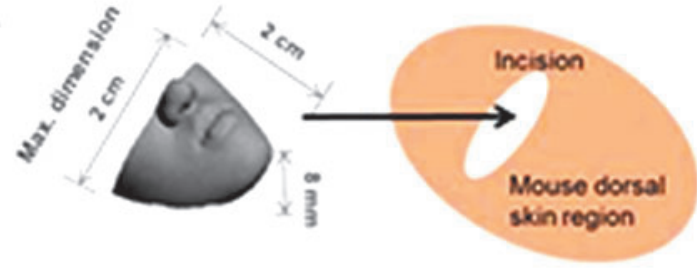

Step 2 - wound creation and BioMask application

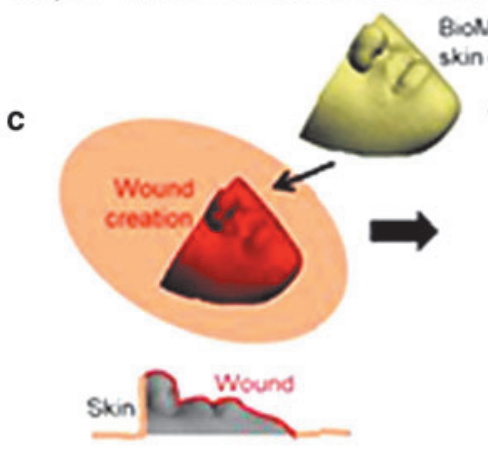

B

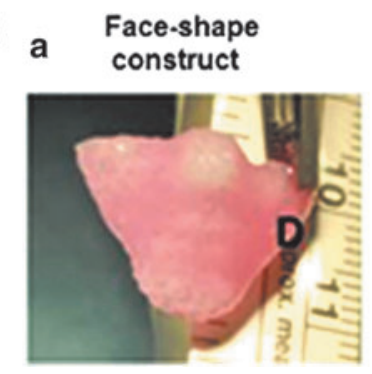

b

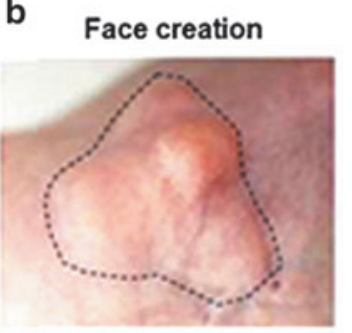

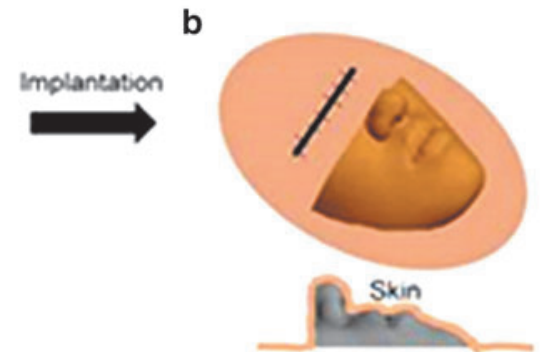

b

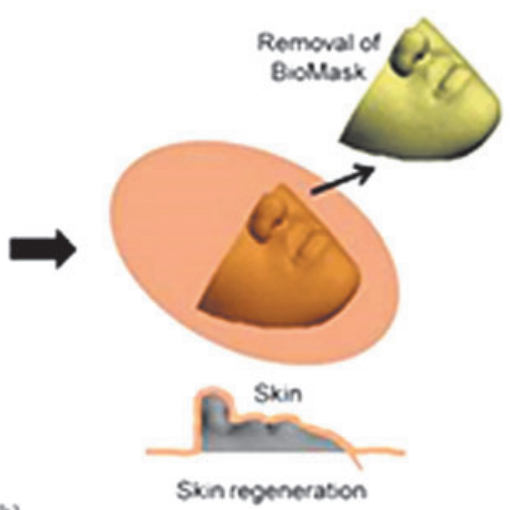

Skin regeneraton

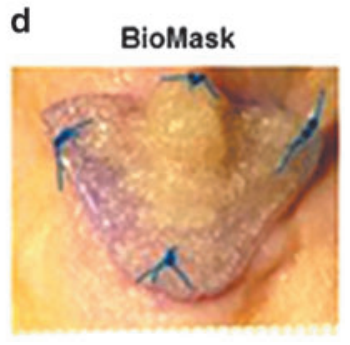

FIG. 1. BioMask implantation. (A) Illustration of skin wound animal model creation and implantation of a predesigned face-shaped architecture. (B) Surgical application of the BioMask. Wound contraction measurement, a reduction on 50\% $(p<0.05)$ of the wound area was observed when the BioMask was implemented. Adapted with permission from Seol et al. ${ }^{36}$ Color images are available online. 
group used in situ bioprinting of autologous skin cells to promote wound healing of full-thickness wounds in murine and porcine models. After excision, full-thickness wounds were covered with a layer of human fibroblasts followed by a layer of human keratinocytes, both suspended in a fibrinogen/collagen-based solution. Excisional wounds showed quick wound closure, diminished contraction, and accelerated re-epithelialization. The regenerated tissue showed commensurate dermal structures and composition similar to that of healthy skin, with extensive mature vascular formation, proliferating keratinocytes, and high collagen deposition in a highly arranged manner. ${ }^{37}$

Apelgren et al. manufactured bioprinted constructs using nanofibrillated cellulose/alginate bioinks mixed with MSCs and adult chondrocytes that were implanted in a subcutaneous pocket in the back of nude mice models. After 45 days, the back-skin pockets were opened, and a fullthickness skin allograft was transplanted and fixated onto the $3 \mathrm{D}$ biofabricated constructs using Prolene sutures. After 60 days, the pocket roof was removed, and the surrounding skin was sutured edge-to-edge to the transplanted skin covering the 3D-printed construct for extra 15 days and engrafted without any macroscopic signs of necrosis or detachment. The results showed that the bioprinted cartilage constructs supported the transplanted skin with nutrients and oxygen delivery, ${ }^{38}$ suggesting that the cartilage bioengineered construct serves as a bed for a full-thickness skin grafts. This approach allows to create a novel 3D setup with potential use on clinical situations when the patient has the need for the reconstruction of composite structures, such as auricular tissues.

\section{Liver}

In late 2018, Mazzocchi et al. combined collagen type-I and hyaluronan (in a ratio of 3:1) to formulate a hybrid bioink to bioprint liver tissue. When cultured with primary human hepatocytes and stellate cells, the tissue showed to be physiologically relevant. The cells maintain their normal urea and albumin production and respond appropriately to acetaminophen (being damaged upon overdosing) for a period of 2 weeks. $^{39}$ To further develop and optimize liveron-a-chip devices, Christoffersson et al. have developed a modified hydrogel embedded with hepatocytes derived from human pluripotent stem cell-derived hepatocytes (hiPS-HEPs). Briefly, they modified hyaluronan and poly(ethylene glycol) hydrogels with cyclic RGD peptides in a perfused chip device to promote the growth, migration, and albumin and urea production of the hiPS-HEPs. ${ }^{40}$

\section{Heart}

Wang et al. have recently assessed the functional properties of the in vitro cardiac tissue model previously developed by the Lewis group. ${ }^{41}$ Briefly, constructs were made of a fibrin-based cell-laden hydrogel (printed together with rat ventricular cardiomyocytes), a gelatin-based sacrificial hydrogel, and poly( $\varepsilon$-caprolactone). They have characterized the cardiac cell synchronization, beating behavior, electrophysiological properties, and contractile force measurement of such constructs and showed a new strategy to bioengineer functional contractile cardiac tissue. These constructs possessed a highly organized structure with the unique physi- ological and biomechanical properties of the native cardiac tissue and exhibited physiologic responses to known cardiac drugs regarding beating frequency and contraction forces. ${ }^{42}$

Noor et al. used an omental tissue biopsy to extract stromal cells and to develop decellularized bioprinted perfusable cardiac patches that entirely match the immunological, cellular, biochemical, and anatomical properties of the patient. ${ }^{43}$ The extracted omental cells were reprogrammed into iPSCs and subsequently differentiated into either cardiomyocytes or ECs. Using the previously described supporting medium method ${ }^{44}$ composed of sodium alginate, xanthan gum, and calcium carbonate, they were able to bioprint functional vascularized patches modeled after the patient's anatomy with elongated cardiomyocytes with actinin striation. ${ }^{43}$

Feinberg's group has recently published an update of the original version of their freeform reversible embedding of suspended hydrogels (FRESH) v1.0 approach, where the authors have engineered 3D collagenous components of the human heart at various scales, ranging from capillaries to the whole organ. ${ }^{45}$ One of the biggest advantages of the FRESH v2.0 is the improved resolution by one order of magnitude, with reliable collagen filaments printed from 200 to $20 \mu \mathrm{m}$ in diameter. The authors developed a porous microstructure to enable rapid cellular infiltration and microvascularization (due to vascular endothelial growth factor [VEGF] incorporation on the bioink), a structure with mechanical strength suitable for the fabrication and perfusion of multiscale vasculature and trileaflet valves. When printed with human embryonic stem cell-derived cardiomyocytes and cardiac fibroblasts, the "artificial" cardiac ventricle showed synchronized contractions, immunofluorescent staining of sarcomeric $\alpha$-actinin-positive myofibrils, directional action potential propagation, and wall thickening of up to $14 \%$ during peak systole mimicking the hallmarks events occurring in health. In addition, they bioprinted whole neonatal human heart that accurately reproduced the anatomical patient-specific features, including the atrial and ventricular chambers, trabeculae, and pulmonary and aortic valves.

In addition to heart models, others have developed microfluidic stroke models with an oxygen scavenging biochip material in combination with integrated opto-chemical oxygen sensing microbeads. These constructs were used to recreate the desired reduced oxygen concentrations and therefore mimic the in vivo conditions during ischemic stroke. ${ }^{46}$ Precise control over oxygen scavenging rates was achieved by variations in material curing protocols (temperatures and curing times), microfluidic layout (higher area to volume aspect ratios), and flow rates. ${ }^{46}$

A recent report has utilized a scaffold-free approach to create tubular cardiac constructs from bioprinted spheroids of clusters formed of iPSC-derived cardiomyocytes, fibroblasts, and ECs. ${ }^{47}$ The authors observed spheroid fusion and beating while still on the needle array. A bioprinter was used to assemble the cardiac spheroids into scaffold-free cardiac constructs, which showed cellular reorganization (troponin T-positive cells on the outer layer of the constructs and CD31-positive cells on the inner regions of the constructs) that may mimic that observed during organ transplantation. This approach is a forward step to overcome the problem of host's immune response to artificial materials, which is a major challenge in cardiac tissue engineering. 
The Lewis group reported a novel biomanufacturing approach named sacrificial writing into functional tissue (SWIFT) that allowed for the manufacturing of organspecific tissues with high cellular densities. The authors used this method to create perfusable vascular channels. To achieve so, they used patient-specific iPSC-derived organoids as "organ building blocks" (embryoid bodies, cerebral organoids, and cardiac spheroids) that were suspended and placed into a collagen-based ECM solution. These structures were thereafter compacted and a sacrificial ink (gelatin-based) written to create the vascularized structures. The results showed that the approach does not affect the intricate microarchitecture of either structure. To further explore this approach, the authors created a functional perfusable cardiac tissue with high cell density composed of organ building blocks of iPSC-derived cells mixed with dermal fibroblasts and the collagen-based ECM. When formed into a small disc-shaped mold, this construct started to beat spontaneously and synchronously with calcium waves that propagate rhythmically and rapidly throughout the tissue. This architecture was then perfused, and over an 8-day period, it developed a pervasive sarcomeric configuration with increased contractility and beating synchronization. Moreover, the authors printed an arterial vascular network geometry within the cardiac organ building blocks matrix, using patient-specific structural data, to accurately replicate the geometry of the left anterior descending coronary artery. ${ }^{48}$ This thorough study opens new windows to create personalized patient- and organ-specific structures with vascular architectures.

\section{Kidney}

Pitsalidis et al. demonstrated recently in a proof-of-principle study that conducting polymers can be used not only as alternative electrode materials but also as electrochemical transistors and simultaneously maintain their scaffolding functions. They demonstrated how an engineered 3D tubularlike system can be used to create a 3D cell culture compartment for barrier integrity studies of 2D kidney barriers under flow conditions. ${ }^{49}$ Using epithelial kidney cells and human fibroblasts cultured on macroporous scaffolds with tuneable properties, the authors showed that cells grow quickly to form tissue-like architectures within the conducting polymer scaffold that constitutes the channel of the transistor. The tissue formation inside the conducting polymer channel progressively modulates the transistor characteristics (configuration). By monitoring the real-time changes in the steady-state characteristics of the transistor with the growth of the cultured tissue, the authors obtained valuable insights on the transients of tissue formation. Moreover, this approach enables label-free, dynamic, and in situ measurements for real-time tracking of 3D cell cultures and compatibility with potential use in long-term organ-onchip platforms.

To provide a tissue-specific microenvironment for renal tissue formation, Ali and Pr have bioengineered an ECMderived hydrogel using decellularized porcine whole kidneys dissolved in acidic solutions and chemically modified by methacrylation. When bioprinted with human renal cells, these structures showed high cell viability, proliferation, and more importantly, cell maturation (into proximal and distal tubular cells and podocytes). When compared with the native renal structures, the artificial architectures showed similar structural (including the formation of tubular and glomerular-like structures) and functional characteristics (electrolyte reabsorption and aminoacid transportation activity). ${ }^{50}$ The authors are currently performing animal model investigations to further characterize this model regarding its interactions with the host tissue.

\section{Skeletal Muscle}

Kim et al. have used a decellularized porcine skeletal muscle tissue as bioink to bioengineer skeletal muscle tissues. The authors modified the decellularized tissue via methacrylation to enhance its mechanical stability and combined it with fibrillated polyvinyl alcohol. Using a previously developed bioprinting method, ${ }^{51}$ the authors fabricated uniaxially aligned fibrillated micropatterned structures. When laden into this architecture, myoblasts were highly aligned with accelerated myogenic differentiated and high degree of myotube formation. ${ }^{52}$ The same group has modified the previous decellularized matrix structures with poly(lactideco-glycolide) (PLGA) to fabricate a multiscale composite scaffold of aligned electrospun ECM fibers and 3D-printed fibrillated PLGA. These composite structures were used to promote aligned cell orientation, differentiation, and myotube formation of human muscle progenitor cells while providing a nanosized basal lamina of ECM. ${ }^{53}$ These two strategies show great potential for applications on musculoskeletal tissue constructs, including tendons and ligaments. In addition, the same group has bioengineered skeletal muscle constructs that showed promising results on muscle function restoration. Briefly, they fabricated a construct composed of a sacrificial acellular bioink, a supporting polycaprolactone pillar, and a fibrinogen/gelatin-based bioink laden with human progenitor cells. The bioprinted tissue showed highly organized multilayered muscle bundles composed of viable, highly packed, and aligned myofiber-like structures. When implanted into rat models with tibialis anterior defects, the constructs showed good integration with the host vascular and neural networks that result in the restoration of muscle formation. $^{54}$

\section{Cartilage}

Cartilage damage is often accompanied with subchondral bone injuries. Therefore, the construction of novel scaffolds that support both cartilage and subchondral bone regeneration appears to be of crucial importance. Li et al. answer that call by coating polycaprolactone scaffolds with a selfassembling peptide hydrogel to promote the proliferation and osteogenic differentiation of rabbit bone MSC and maintain the chondrocyte phenotype. ${ }^{55}$ When implanted in vivo, these structures induced simultaneous regeneration of cartilage and subchondral bone.

Multicellular spheroids of chondrocytes, ECs, and MSCs were bioprinted in a scaffold-free approach to generate artificial tracheas. These were matured in a bioreactor for 28 days and then transplanted into rat animal models as a tracheal graft. The bioprinted artificial trachea showed sufficient mechanical strength to be transplanted into the trachea of rat models, with chondrogenesis and vasculogenesis being observed as soon as 8 days post-transplantation. ${ }^{56}$ 
Several strategies have been recently developed to increase the viability of chondrocytes. Mekhileri et al. have developed a two-step approach to achieve enhanced cell viability by constructing a bioassembly system that consisted of a fluidic-based singularization and injection module incorporated into a 3D bioprinter apparatus. This singularization device collects the pooled microtissues from the high-throughput fabrication process to then deliver individual microtissues to an injection module that allows for their insertion into precise locations within a 3D-plotted scaffold. This method was used to fabricate bilayered constructs by alternatively depositing a thermoplastic polymer solution and inserting predifferentiated chondrogenic microtissues or cell-laden gelatin-based microspheres. ${ }^{57}$ In addition to long-term chondrocyte viability, these constructs also showed deposition of cartilage-specific ECM proteins, and clinically relevant shape and size. The flexibility of this approach could also be used to pick and place cellular spheroids in a priori designed locations and used to study specific pathological conditions in a 3D in vitro model system.

Malda's group has bioengineered microfiber-reinforced hydrogels that simultaneously capture the zonal depthdependent mechanical properties of native cartilage and support neocartilage formation. ${ }^{58}$ By combining melt electrospinning of poly( $\varepsilon$-caprolactone) with a gelatinmethacrylamide hydrogel, the authors developed a bilayered microfiber architecture of a densely distributed crossed fiber mat (superficial tangential zone) to improve the load-bearing properties of the construct and a uniform box structure (middle and deep zone). The acellular composite structure showed a stress relaxation response comparable with the tested native cartilage, and when cultured with chondrocytes under mechanical conditioning, significant production of sulfated glycosaminoglycans and collagen type II was observed. For the first time, the authors showed the importance of incorporating a viable superficial tangential zone in articular cartilage tissue engineering (TE) applications.

\section{Bone}

Bone defect repairs are still challenging clinical problems in musculoskeletal systems. Lai et al. have addressed these problems by developing a novel porous scaffold composed of magnesium, poly (lactide-co-glycolide), and $\beta$-tricalcium phosphate. These were deposited using low-temperature AM and transplanted into rabbit models with femora condyle defects. At 12 weeks postsurgery, histological and mechanical properties were assessed, and a significant new bone formation and newly formed vessels with well-architected structure were observed. In addition, these constructs showed well-designed biomimic structures and improved mechanical properties, making them a promising composite biomaterial for repairing challenging bone defects. ${ }^{59}$

Graphene oxide was used to improve the structural stability and osteogenic properties of alginate-based bioinks. Choe et al. used such composites bioprinted with MSCs to enhance their osteogenic differentiation. ${ }^{60}$

To efficiently promote osteogenic differentiation of adult stem cells, Wei et al. developed silk fibroin-based 3Dbioprinted composite (together with gelatin, hyaluronic acid, and tricalcium phosphate) coated with human platelet-rich plasma. ${ }^{61}$ Human adipose-derived MSCs cultured on such structures significantly upregulated the gene expression levels of late osteogenic markers and showed enhanced growth and proliferation profiles. This showed to be a more efficient approach to drive osteogenic differentiation of adult stem cells with potential applications for bone TE.

The variety of materials combined with the low costs associated with extrusion-assisted bioprinting (reviewed recently by Miri et $a l^{62}$ ) has made it the primary choice to fabricate bioprinted microfluidic platforms. However, the resolution is limited to roughly $30-100 \mu \mathrm{m}$ for the current bioink systems, and therefore, optical-based techniques have been developed to provide better resolutions.

\section{Other Biofabrication Techniques-Optical-Based Techniques}

In extrusion-assisted AM, a material is dispensed through an extruder or nozzle while a computerized arm moves the nozzle to create the 3D shape. Direct extrusion AM of microchannels can be challenging due to the collapse of the top layers into the microchannels; therefore, this technique is often combined with a support material that is removed postprinting (sacrificial printing). The selective removal of the sacrificial material leads to a hollow microfluidic system in the core structure. In addition to extrusion-assisted AM, recent approaches have focused on light reactions to solidify the structure and make microchannels with higher resolution. ${ }^{63}$ As there are no nozzles being used during fabrication, clogging is not an issue, so higher viscosity fluids could be processed, and there is no risk of shear stress-induced cell damage or phenotype alterations. When compared with the conventional extrusion-assisted AM, photopatterning shows higher resolution and better cell viability. ${ }^{64-66}$ One remaining challenge in optical-based bioprinting is the synthesis of a larger palette of hydrogels that can better mimic the ECM properties, thus interacting more and more with the encapsulated cells. Here, we classify the optical-based bioprinting technique to three main technologies: SLA, LIFT, and two-photon lithography.

\section{Stereolithography}

In conventional SLA apparatus, a laser beam raster scans across the liquid resin or hydrogel solidifying it through a photochemical reaction. ${ }^{16,67}$ SLA does not require the use of expensive molds, which may lead to an efficient commercialization of the organ-on-chip platforms manufactured using this technique. ${ }^{62}$ An extensive review on fabricating tissue scaffolds using photopolymerization with particular focus on SLA approaches was recently published by Whittington's group. ${ }^{67}$ In a staggering study, Grigoryan and Paulsen exploited the use of food dyes as photoabsorbers in photo-crosslinkable hydrogels to increase the resolution and diminish the light diffusion in their constructs. ${ }^{68}$ Their fabricated intravascular and multivascular bioprinted constructs, perfused with red blood cells, showed capacity for oxygenation and functionality in vivo in a hepatic model. However, the photo-crosslinkable hydrogel itself was not loaded directly with cells during fabrication. By doing so, they reduced the light diffusion during the process, increasing the resolution and the complexity of the final construct. ${ }^{68}$ As a result, they could fabricate intricate structures 
to study the oxygenation of red blood cells in an alveolarlike vascular network. These vascular structures were used to explore the oxygenation of alveolar airways and chronic liver injuries.

Grix et al. fabricated liver equivalents for advanced organ-on-a-chip applications using SLA with two bioinks based on gelatin and polyethylene glycol. Briefly, they fabricated hexagonal constructs with 12 hollow channels to allow for perfusion of complete organoids. These constructs were incorporated with HepaRG cells (cells able to differentiate toward the biliary-like and hepatocyte-like phenotype) and human hepatic stellate cells. The bioprinted liver tissue equivalents were found to have high albumin and cytochrome $\mathrm{P}_{450} 3 \mathrm{~A} 4$ (a liver enzyme) expression, tight junction protein ZO-1 and MRP2 (a liver-specific bile transporter multidrug resistance-associated protein 2) expression, and the overall metabolism remained stable with normal levels of glucose, lactate, and lactate dehydrogenase production. ${ }^{69}$

Parrish et al. have used SLA to construct a dual perfusion microphysiological bioreactor platform into a 96-well plate to support a large number of samples and recreate the parenchymal barrier tissue constructs, while maintaining sample addressability and compatibility. Using such approaches, they developed a (1) novel perfused biofabricated 3D ovarian cancer spheroid model with anticancer drug screening capabilities and (2) a perfused bioprinted vascular coculture model of human umbilical vein endothelial cells (HUVECs) and bone marrow-derived MSCs. In addition, these constructs showed the capacity to support nondestructive quantitative computed tomography imaging for soft and hard tissue. ${ }^{70}$

Huebsch et al. developed a heart-on-a-chip device for metabolically driven maturation of human iPSC (hiPSC)derived cardiomyocytes. They constructed polydimethylsiloxane (PDMS) devices using two-step photolithography to build a 3D cardiac microphysiological system to improve immediate microtissue alignment and tissue-specific ECM production. Through the 3D structures, cells showed changes regarding their electrophysiology and pharmacology, which were not observed in the 2D monolayer models of the same cell type. Cell contraction was measured via PDMS micropillar deformation and correlated with gene expression to observe the systematic combination of biophysical stimuli and metabolic cues, and therefore improve the electrophysiological maturation of hiPSC-derived cardiomyocytes. ${ }^{71}$ Following similar approaches, Caluori et al. have combined an electrochemical multielectrode array with atomic force microscopy measurements to record the beating rates of cardiomyocyte organoids together with the deformation of the cardiac cluster during contraction following effects of heart drugs. ${ }^{72}$

To improve the outcomes from bioprinted muscle equivalents, Shima et al. have developed a stretchable culture system for muscle-like constructs. Briefly, molds for the culture stages, stamps, and anchors were fabricated by SLA using PDMS, and myocytes laden into a collagen/Matrigelbased bioink. After plating, the hydrogel containing myocytes was molded with the PDMS stamp and posteriorly fixed using anchors. These structures were either left unstimulated or electrically stimulated to induce muscle contraction. The authors observed the formation of multi- nucleated myotubes with sarcomere formation and intercellular calcium transfer, necessary for muscle contraction. Despite showing spontaneous contraction on nonstimulated structures, it appears sporadically and not synchronized in the tissue. When electrically stimulated, a second interval contraction rate was detected. ${ }^{73}$ These architectures pave new routes for new insights on basic biology research and drug screening for skeletal muscle TE applications.

\section{Volumetric Bioprinting}

The scalability of AM techniques is limited by their printing velocity since lengthy biofabrication processes may impair cell viability and proliferation. To overcome these limitations, Bernal et al. have bioprinted clinically relevant sized and anatomically shaped structures in a matter of tens of seconds by using volumetric bioprinting. Using gelatinbased photoresponsive hydrogels and optical-tomographyinspired printing approaches (digital light projection printing using digital micromirror devices), the authors have created living tissue constructs of a human auricle (Fig. 2) and trabecular bone models that promote high cell viability. In addition, the volumetric bioprinting structures showed artifactfree surface features, reproducing more faithfully the surface features of the model particularly when compared with other AM approaches (Fig. 2D), and the possibility to bioprint free-floating parts without the need for sacrificial ink support materials to therefore generate structures that can reversibly modify their shape postprinting as often applied in $4 \mathrm{D}$ bioprinting. ${ }^{74}$ The authors reported a novel biofabrication strategy to rapidly create large structures that can be potentially loaded with high cell numbers for regenerative medicine applications.

\section{Laser-Assisted Bioprinting or LIFT}

Recent studies have shown that this technique is the least productive regarding the volume of bioprinted material per minute. In addition, the preparation of samples is costly and demanding. It also requires extremely high percentages of relative humidity in the bioprinter enclosure to counter effect cell dehydration. ${ }^{75}$ This approach, on the contrary, makes it possible to manufacture structures with cell-level resolutions, and the bioink can be loaded with cell densities comparable with those observed on living tissues. ${ }^{76}$

In an innovative approach, Kérourédan et al. combined endothelial progenitor cells (EPCs) with stem cells from the apical papilla (SCAPs) as osteoprogenitors to promote bone formation and induce the formation of a vascular network. They deposited specific high cell density patterns of EPCs, using laser-assisted bioprinting onto a collagen hydrogel previously cultured with SCAPs. They observed the formation of capillary-like networks at day $6 .{ }^{77}$ On a follow-up study using murine models, the same group investigated the deposition of ECs in situ in calvaria defects to promote bone regeneration. ${ }^{78}$ The bone calvaria defects were prefilled with collagen hydrogels preloaded with MSCs prior the deposition of a specific EC pattern. They observed, 2 months postimplantation, significantly higher rates of vascularization and bone regeneration when ECs are cultured in specific patterns and densities than when seeding randomly, ${ }^{78}$ corroborating the importance of patterning on vascularization and bone regeneration. 
A

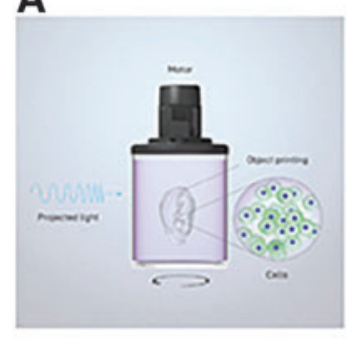

D Volumetric printing

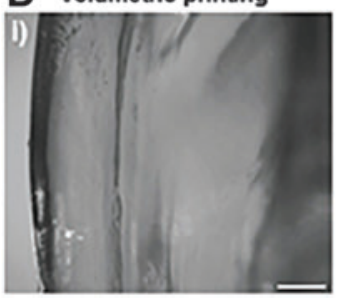

B

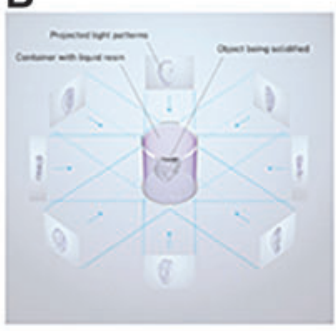

Extrusion-based

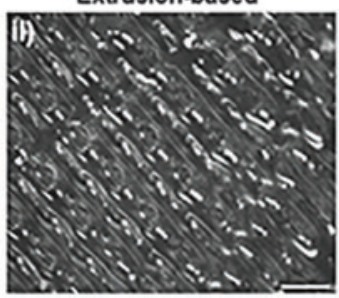

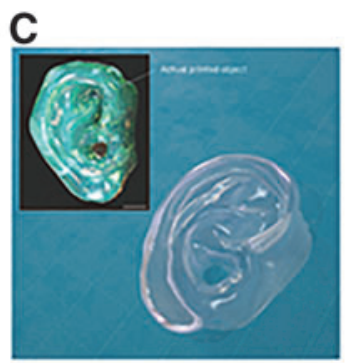

Digital Light Processing

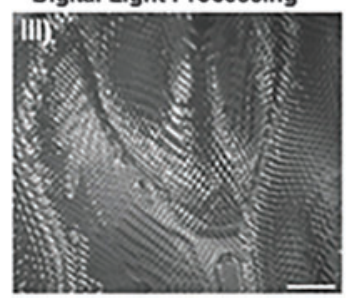

FIG. 2. Volumetric bioprinting process. (A) The cell-laden reservoir is connected to a rotating platform, (B) tomographic projections of the human auricle model, and (C) resulting printed architecture. (D) Comparison of the surface features of the fabricated auricle through volumetric printing, extrusion-based printing, and digital light processing (scale bar: $500 \mu \mathrm{m}$ ). Adapted from Bernal et al. ${ }^{74}$ Color images are available online.
Sorkio et al. have produced three types of human corneal structures using recombinant human laminin and human sourced collagen I as the bases for the functional bioinks. ${ }^{79}$ The stratified corneal epithelium mimicked using human embryonic stem cells, while the lamellar corneal stroma was produced by alternating acellular layers of solely bioink and layers with human adipose tissue-derived stem cells. The bioprinted cornea-mimicking tissues showed to promote cell proliferation and viability, morphological features resembling those of the human ocular epithelium, expression of key markers of differentiated corneal epithelium, and formed organized corneal stromal-like structures. In addition, when transplanted into porcine organ cultures, the 3Dbioprinted stromal structures attached to the host tissue and histological observations showed migration of the adipose tissue-derived stem cells into the host corneal stroma. This is the first study to demonstrate the feasibility of 3D laserassisted bioprinting for corneal applications. The same group has compared the behavior of hiPSCs when these were laser bioprinted in combination with different biomaterials. The authors have suggested that the cells are effectively more sensitive to the applied biomaterials, but not to laser bioprinting per se, regarding their viability, proliferation, and differentiation potential. ${ }^{80}$ The results showed that the combination of bioprinted hiPSCs with hyaluronic acid with pluripotency supporting cell culture medium on $\mathrm{Ma}-$ trigel allowed the generation of precise predefined hiPSC patterns that maintain their pluripotency and differentiation potential. This is the first successful approach to bioprint hiPSCs by laser-based techniques, with huge potential to be applied when highly controlled bioprinted patterns without interfering with cell pluripotency are required.

Kim et al. have created a silk fibroin-based bioink by a methacrylation process for digital light processing. These fibroin-based hydrogels showed outstanding mechanical, rheological (constant storage and loss modulus upon shear strain), and cytocompatibility properties that are adequate for experimental testing with potential application in various TE structures. When incorporated with human chondrocytes and bioprinted into a trachea-like shape, these architectures showed cartilage-like features, including cell organization and a matrix rich in proteoglycans and collagen. ${ }^{81}$ This approach showed the potential applications for a naturally occurring polymer to be used as bioink with equivalent strength and stability properties when compared with most common alternatives, which are often combinations of natural and synthetic materials.

\section{Two-Photon Polymerization}

2-PP is a lithography-based AM technique that enables the biofabrication of structures with submicrometer resolution. ${ }^{82}$ An extensive review on materials, techniques, and appropriate geometries to establish a conceptual framework to engineer biological constructs with 2-PP has been recently published elsewhere. ${ }^{83}$ Pennacchio et al. have fabricated instructive gelatin-based building blocks using 2-PP for the production of anisotropic collagen microtissues in bottom/up tissue engineering. The linear topography strongly enhanced cell alignment and production of oriented collagen fibers into highly anisotropic microtissues. ${ }^{84}$

Ovsianikov's group has, over the last year (or so), provided valuable developments on 2-PP technology. ${ }^{85}$ Using a gelatin-norbornene-based bioink, previously developed by the same group in Van Hoorick et al., ${ }^{86}$ embedded with fibroblasts, the authors showed that these constructs had outstanding biocompatibility, supported cell adhesion, migration, and proliferation. In addition, the direct embedding of cells into the porous scaffold provided a uniform cell distribution and high cell loading capacity independently of the pore size. ${ }^{82}$ This was the first study that showed the possibility of producing cell-embedding hydrogel constructs manufactured by 2-PP technologies, paving the way to new high-resolution bioprinting.

\section{Laser-Induced Hydrogel Degradation}

One of the biggest challenges when generating large constructs for TE is the ability to recapitulate the dense and complex in vivo vascular networks. To overcome this problem, Slater's group has developed an image-guided, laser-induced hydrogel degradation approach that uses synthetic networks or 3D image stacks of in vivo vasculature as 
templates to fabricate complex microvascular networks. ${ }^{87}$ In a follow-up study, the same group has developed an automated method to design synthetic augmented channels to enforce controlled flow properties within the 3D networks. Using computational fluid dynamics methods, they demonstrated flow predictability that closely matches the experimental results, ${ }^{88}$ opening a new door to create complex networks that mimic the in vivo counterparts.

\section{D Bioprinting-A New Direction}

The recent advancements in the fields of microfluidic and tissue engineering have opened the doors to design and construct new in vivo models of several diseases. Despite attracting a great deal of attention, several limitations are found in 3D bioprinting technologies. Remaining challenges include the ability to fabricate proper hollow constructs, ${ }^{89}$ to develop functional tissue vascular constructs with multiscale vasculatures, ${ }^{90}$ and to provide the adequate microenvironment for cell growth, proliferation, and differentiation in thick 3D structures. ${ }^{90}$ When time enters the equation of 3D bioprinting as the $4 \mathrm{D}$, the shapes and functionalities of the bioprinted constructs can change over time under external triggering, leading to the arise of 4D bioprinting. ${ }^{91}$ Broadly, 4D bioprinting includes (1) the deformation of the materials' shape (conventional or smart materials) and (2) the maturation of the engineered constructs in a time-dependent process. ${ }^{91}$ Although the latter proves to be a promising strategy to overcome the challenges of $3 \mathrm{D}$ bioprinting and better recapitulate the native tissue, ${ }^{92}$ it is a general principle of any tissue engineering process. Hence, 4D bioprinting is more properly addressed when there is an active timedependent change of the biological constructs, which results in enhanced functionality beyond the conventional tissue maturation. Therefore, this technology offers the possibility to create immense varieties of structures with the highest complexity and resolution.

Shape transformation in 4D biofabrication can be categorized into three main approaches. The first includes shape transformation of acellular constructs to then load those with cells. This is comparable with the traditional scaffoldbased approach, and less-attractive once living cells restrict the selection on the materials to use and fabrication method. The second approach includes the deposition of cells onto a printed construct, and shape transformation of the already cell-loaded construct, which is an appealing strategy to provide structural and surface time-dependent instructiveness to the seeded cells. The third approach is the fabrication of a structure with a nonvital material and encapsulation of cells simultaneously, followed by its shape transformation. This shows to be a challenging method due to the practical limitations on controlling cell deposition during the bioprinting process. Regardless, the latter two approaches have huge advantages as the shape transformation occurs while cells have already been incorporated into the construct. The physical shape transformation can be triggered by various stimuli, which lead to the shape morphing of the object, either manually or using the so-called, smart materials with shape-changing properties. ${ }^{92}$ These triggering agents can be categorized into four main approaches: (1) stimuli responsive, (2) spontaneous shape transformation, (3) cell contraction, and (4) manual transformation. ${ }^{92}$
Stimuli-responsive processes can be triggered by moisture, temperature, $\mathrm{pH}$, magnetic and electric fields, and light. ${ }^{90}$ This approach offers several advantages over the other methods, as it allows for the precise control of the moment when shape transformation is needed. It also allows for the folding of multiple objects made of different materials at multiple scales. Temperature and water sorption stimulation are the most common shape transformation factors. Few studies published before 2018 have extensively addressed these two stimuli approaches. ${ }^{91,93,94}$

For the first time, Su et al. fully explored the 4D printing potential of a commercial self-morphing polymer, SU-8, previously developed by the same group. ${ }^{95,96}$ They allowed the polymers deformed temporally to be fixed by vitrification or crystallization of the polymer chains, and to return to their original shape under an external stimulus through spatial control of the swelling medium (cyclopentanone) inside the polymer matrix. ${ }^{96}$ By doing so, the authors created passive and active regions within the polymers that control the self-morphing processes to form different geometries following stimuli. As a proof-of-concept, they showed that a soft actuator can switch functions to an electrical circuit. These two studies help paving new routes for the exploration of other potential candidates, as SU-8 polymer is usually utilized as a photoresist and not designed to fabricate smart structures for biomedical devices.

With applications on soft tissue injuries, Zhang et al. have constructed a magnetic-responsive drug delivery dressing using tetra-PEG/agar hydrogels incorporated with $\mathrm{Fe}_{3} \mathrm{O}_{4}$ nanoparticles. In vitro and in vivo drug release studies in response to changes on the tuned magnetic field showed better performance of this system when compared with commercially available ointment systems. ${ }^{97}$ This magneticresponsive hydrogel showed to be a good candidate to improve recovery of injury on soft tissues.

Betsch et al. have also incorporated iron nanoparticles on printable bioinks to generate complex multilayered tissues. By applying a straightforward magnetic-based mechanism in hydrogels during bioprinting, they constructed aligned collagen fibers using less concentrated hydrogel blends. Aiming to apply such constructs for cartilage TE, the authors bioprinted constructs with alternated layers of aligned and random fibers. The cell-loaded constructs with alternating layers of aligned and random fibers expressed significantly more collagen II in comparison with the solely randomly oriented fiber constructs. ${ }^{98}$ These observations corroborate the importance of the structural and architectural properties of bioinks used in bioprinting for their use in tissue engineering applications.

Miao's group has fabricated biomimetic hierarchical 4D micropatterns with smart soybean oil epoxidized acrylate bioinks, using a unique photolithographic-stereolithographictandem strategy. The distinct topographical surfaces of these bioengineered structures and the external stimulus, delivered immediately after fabrication printing, effectively regulate the cardiomyogenic behaviors of human bone marrow MSCs. The MSCs readily grew and were highly aligned along the micropatterns, forming uninterrupted cellular sheets. ${ }^{99}$ Upon external stimuli, a 4D dynamic shape change from 2D designs into a flower-like structure was observed. The printed scaffolds also possessed a shape memory effect beyond the $4 \mathrm{D}$ features. A proof-of-concept 4D patch for cardiac 
regeneration was for the first time shown, and the fabricated patch demonstrated significant cardiomyogenesis potential suggesting its promising potential for tissue and organ regeneration applications. ${ }^{99}$ The same group has also fabricated a 4D anisotropic skeletal muscle tissue using a "staircase effect" strategy and investigated the effects of topographical cues on the skeletal muscle differentiation of human bone marrow MSCs. ${ }^{100}$ Using a layer-by-layer coating together with a shape memory polymer, smart constructs exhibiting shape fix and recovery processes were prepared. The topographical cues of such constructs significantly increased the expression of myogenic genes, suggesting their application potential for tissue engineering constructs, including skeletal muscle. The same group has additionally developed 4D electroresponsive biomaterials that showed huge potential for nerve tissue regeneration applications. Multiresponsive graphene hybrids with 4D-printed architectures were created, which provided numerous nerve regeneration characteristics, including physical guidance, chemical cues, and seamless integration. ${ }^{101}$ This stimuliresponsive $4 \mathrm{D}$ technique combined with the previous bone tissue fabrications may pave new ways for repairing bone defects that are accompanied by nerve damage.

Parameswaran-Thankam et al. have fabricated injectable thermoresponsive hydrogels of hydroxypropyl guar-graftpoly(N-vinylcaprolactam) modified with nanohydroxyapatite with bone tissue engineering applications. The graft copolymer showed excellent thermogelling and injectable properties suitable for in vitro osteoblast differentiation, controlled drug delivery of ciprofloxacin, and apatite-like structure formation. ${ }^{102}$ Thermoresponsive materials have also been modified with hyaluronic acid and chitosan to fabricate novel injectable scaffold with osteoinductive and osteoconductive activities to support the formation of newly vascularized bone. ${ }^{103}$ Briefly, graphene oxide was combined with a thermoresponsive hydrogel to support the survival and proliferation of MSCs and induce the early bone-forming marker alkaline phosphatase and osteogenic regulator and bone marker expression by MSCs. When transplanted in vivo well-mineralized and highly vascularized trabecular bone was observed. Similar studies were more recently published by Vojtova et al., ${ }^{104}$ where thermoresponsive materials are further modified to fabricate an injectable hydrogel for bone tissue regeneration.

\section{Bioassembled In Vitro Tissue Models}

As previously mentioned, bioassembly involves the integration of various-shaped cellular building blocks to reconstruct organomimetic macroscopic cellular tissues. An extensive review on the different bioassembly techniques and produced structures has recently been published. ${ }^{2} \mathrm{We}$ therefore focus solely on the recent research articles published in this field.

Layer-by-layer polylactic acid membranes were fabricated by FDM. These constructs were seeded with cocultures of human bone marrow stromal cells and EPCs to improve vascularization in vivo. Four cellularized membranes were then assembled in a layer-by-layer manner and early osteoblastic and EC differentiation markers, such as alkaline phosphatase and von Willebrand factor, were expressed in all layers of the assembly in a homogenous manner. When implanted subcutaneously in vivo in mice models, these constructs showed high degree of vascularization. ${ }^{105}$ This approach showed to be an efficient method to obtain a homogenous cell distribution and blood vessel formation within an entire volume of a $3 \mathrm{D}$ composite scaffold.

A novel clamp-shaped micromachine was developed by $\mathrm{Li}$ and Wang using nickel nanoparticles for the indirect untethered bioassembly of cell-laden micromodules. Using a multilayer template, the magnetic nanoparticles were mixed with PDMS for mold replication of the micromachine with a high-resolution and permeability. The hydrogel microstructures (poly(ethylene glycol) diacrylate modified with RGD peptides) loaded with mouse fibroblasts were effectively assembled into microtissues with precise reconfigurable shape and composition. These constructs were modified to mimic blood vessels, by making a hole in the center of the micromodule. ${ }^{106}$ Results showed that indirect magnetic manipulation is an efficient and versatile bioassembly method for cellular micromodules, and the high level of cell viability makes these structures of potential interest for drug screening.

Mi et al. have constructed a liver sinusoid on a chip device using self-assembly of ECs and hepatocytes. ${ }^{107}$ By synchronously injecting two kinds of cell-laden collagens, hepatic-laden and endothelial-laden collagen, they formed two distinct collagen layers with a clear boundary. This construct showed high levels of cell viability, and physiological relevant properties in terms of albumin and urea production. In addition, acetaminophen treatment confirmed that only a high dose of the drug for a long period would cause acute liver injury, and a low dose did not lead to an obvious death of hepatocytes. These results showed that this liver sinusoid mimic supports hepatocyte viability and functionality and is beneficial for drug screening.

Flegeau et al. have fabricated a model of kidney glomerular barriers using bioassembled podocytes and ECs. ${ }^{108}$ Briefly, shells of $2 \%$ reticulated alginate were formed around a polycarbonate capillary tube and ECs suspended in a collagen I solution were injected inside the capillary tube to form a glomerular microfiber (Fig. 3A-a-c). Podocytes were dropped onto the fibers to form a glomerular barrier. These constructs showed high cell viability (Fig. 3A-d) and expression of specific proteins (including VEGF receptor 2 and von Willebrand factor) (Fig. 3B). More importantly, they showed ultrastructure similarities to those of human glomerular barrier.

\section{Conclusion and Future Outlook}

AM commercial applications at large are unceasingly expanding and its market size is expected to reach $\$ 10.8$ billion by $2021,{ }^{109}$ with several companies already working on bioprinted products for TE applications, including cartilage, liver, and bone tissue. ${ }^{110}$ Here, we have revised the efforts in the past couple of years to clarify the confusion in terminology and concepts on biofabrication. We then highlighted the major evolutions on 3D and 4D bioprinting of tissues. However, several future developments can be expected. The biggest drawbacks of large-scale cell-based therapies are the limited oxygen and nutrient supply to the innermost parts of the bioprinted structures and the efficient 
FIG. 3. (A) The cellularized glomerular microfiber. (a) The cellularized microfiber was contained in the shell formed of successive layers of reticulate alginate.

Glomerular microfibers kept a similar conformation and structure to the native tissue after (b) $18 \mathrm{~h}$ and (c) 5 days upon alginate shell removal. (d) Endothelial cells show high viability (scale bar: $500 \mu \mathrm{m}$ ). (B) Glomerular microfibers showed the expression of specific endothelial markers at day 14 (immunostaining of (A) PECAM-1, (B) vWF, (C) Nephrin, (D) Synaptopodin). Blue arrows show the Weibel-Palade bodies (scale bar: $50 \mu \mathrm{m}$ ). Adapted from Flegeau et al. ${ }^{108}$ Color images are available online.
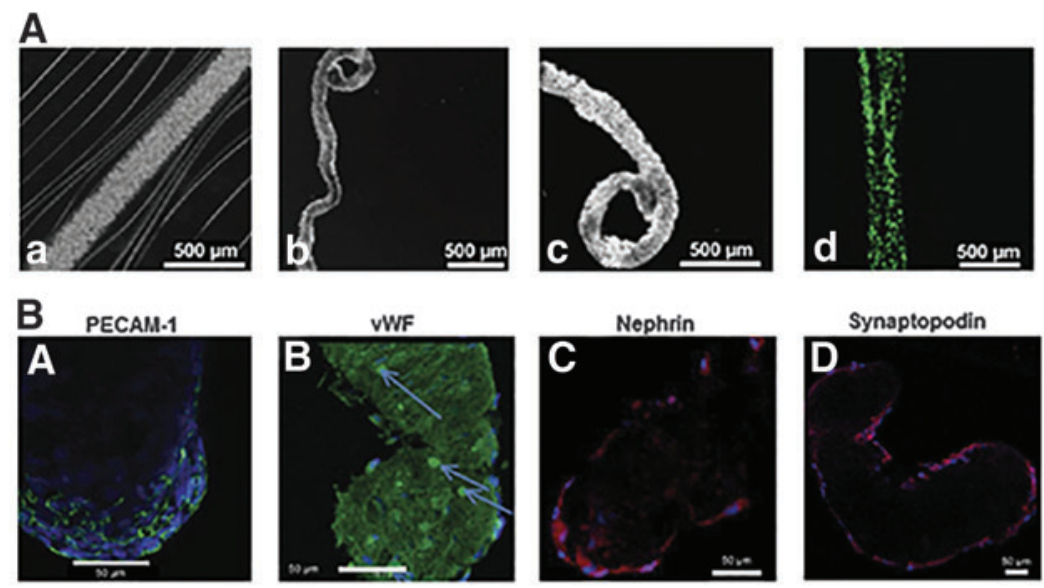

waste product elimination. Vascular trees composed of large vessels and capillaries are then required for the correct maintenance of large tissues. However, limitations in bioprinting resolution and speed are still big challenges to bioprint vascular structure, as the highest resolutions, currently achievable with laser-based bioprinters, is one order of magnitude lower than the size of a human capillary. Therefore, we postulate that the construction of perfusable branched systems with increasingly smaller microvessels is of upmost need in a timely manner to prevent tissue necrosis. Several attempts to enhance angiogenesis on 3Dbioprinted constructs have been recently reported and extensively reviewed by $\mathrm{Ke}$ and Murphy. ${ }^{111}$ Despite their encouraging results, these strategies still need refinement. In addition to vascular trees, the development of an innervation network within the constructs is also fundamental for the normal function of tissues. However, this would be easier to achieve as it may be inducible using pharmacologic or growth factor signaling approaches. Regarding the engineering of neural tissues, the main hurdle arises from the complexity and difficulty of accurately replicating the complex structures of the neurovascular axis. Recent investigations (although before 2018) have partially tackled this issue by bioengineering a blood/brain barrier with a high degree of complexity $^{112}$ as an example. In addition to the fabrication of higher complexity structures, functionalization of bioinks with basal lamina ECM components may also aid on recapitulating the complex cellular responses in neural tissues.

One big challenge in AM is the fabrication of sufficiently stable and mechanically rigid constructs to ensure successful transplantation. This is of special concern in hard tissue applications as the elastic modulus of the bioprinted structures needs to be high to maintain their physical structure while maintaining the porosity to support normal cell growth and viability.

Several studies presented in this review have applied their bioengineered approaches into in vivo animal models. However, some have tested their constructs in immunocompromised models, which lack the appropriate information regarding the inflammatory response and foreign body reaction of the host animal to the artificial constructs. Therefore, implanting these structures into preclinical immune-competent animal models is of upmost need. Prompting the integration of bioengineered tissues by the host model also assumes a critical factor for the clinical success of these approaches.
After bioprinting, tissues require maturation for cell assembly and growth. Bioreactors may provide the necessary maturation factors and other physiological relevant factors for preimplantation testing of the constructs. The choice on the correct maturation times, addition of mechanical loading, and cell culture media and its supplements (particularly the use of animal-derived factors) are urgent issues to be addressed regarding the potential clinical applications of these approaches.

Virtually there are no limitations on the choice of polymers to be used in AM approaches, and more studies similar to the ones of Su et al. ${ }^{96}$ where a commercial polymer was used in a completely different context than what originally designed for, to fabricate smart structures for biomedical devices, are needed. By applying the same rationale, one would broaden the possibilities to explore new potential candidates with applicability on biofabrication. A recurrent question in bioprinting remains if multiple materials can be deposited within the same process to further mimic the complex heterogeneity of the living tissues. By doing so, material gradients, replication of biological interfaces, and vascularization could all be introduced in one single step. Whether this can be achieved by extrusion and inkjet bioprinting, optical-based bioprinting remains limited from this perspective. By interconnecting different bioprinted tissues for drug screening prior to starting clinical trials could eventually reduce, or perhaps even eliminate, the use of animal models. Following the steps of Skylar-Scott et al. ${ }^{48}$ we postulated that by using gene-edited cells derived from the patients to be laden into 3D structures, one can achieve a more functional tissue equivalent with closer properties to those of the native tissue.

As the "next-generation" biofabrication technique, 4D bioprinting still faces several limitations that remain to be overcome. The use of biocompatible biomaterials that allow to obtain high cell density while maintaining the appropriate rheological properties is still an unmet property of the smart materials. However, new approaches are now being developed to meet the desired properties, which include the use of hybrid structures to respond to multiple stimuli. To closely mimic the nature of tissues and organs, precise stimulation methods and high-resolution multimaterial bioprinters are also necessary.

3D and 4D bioprinting has taken significant leaps in several technological aspects that are leading TERM to 
higher levels of tissue and organ bioengineering complexity. Not only faster fabrication of engineered constructs has been achieved, but structures with high complexities and resolutions are also being tailored and manufactured. The success of TERM-based therapies always relies on the development of accurate and reliable diagnostic tools based on the anatomical and physiological characteristics of the patient. 3D and 4D bioprinting has a large potential to impact TERM research toward more personalized and accurate approaches that can be more easily translated into clinic.

\section{Disclosure Statement}

No competing financial interests exist.

\section{Funding Information}

We are grateful to the European Research Council project "Cell Hybridge" (grant no. 637308).

\section{References}

1. Groll, J., Boland, T., and Blunk, T. Biofabrication: reappraising the definition of an evolving field. Biofabrication 8, 013001, 2016.

2. Moroni, L., Burdick, J.A., and Highley, C. Biofabrication strategies for 3D in vitro models and regenerative medicine. Nat Rev Mater 3, 21, 2018.

3. Moroni, L., Boland, T., and Burdick, J.A. Biofabrication: a guide to technology and terminology. Trends Biotechnol 36, 384, 2018.

4. Groll, J., Burdick, J.A., and Cho, D.W. A definition of bioinks and their distinction from biomaterial inks. Biofabrication 11, 013001, 2018.

5. Weisgrab, G., Ovsianikov, A., and Costa, P.F. Functional 3D printing for microfluidic chips. Adv Mater Technol 4, 1900275, 2019

6. Gomes, M.E., Rodrigues, M.T., and Domingues, R.M.A. Tissue engineering and regenerative medicine: new trends and directions-a year in review. Tissue Eng Part B Rev 23, 211, 2017.

7. Harrison, R.H., St-Pierre, J.P., and Stevens, M.M. Tissue engineering and regenerative medicine: a year in review. Tissue Eng Part B Rev 20, 1, 2014.

8. Park, K.M., Shin, Y.M., and Kim, K. Tissue engineering and regenerative medicine 2017: a year in review. Tissue Eng Part B Rev 24, 327, 2018.

9. Fritz, M., Belcher, A.M., and Radmacher, M. Flat pearls from biofabrication of organized composites on inorganic substrates. Nature 371, 49, 1994.

10. Fong, H., White, S.N., and Paine, M.L. Enamel structure properties controlled by engineered proteins in transgenic mice. J Bone Miner Res 18, 2052, 2003.

11. Wu, L.Q., and Payne, G.F. Biofabrication: using biological materials and biocatalysts to construct nanostructured assemblies. Trends Biotechnol 22, 593, 2004.

12. Mironov, V., Trusk, T., and Kasyanov, V. Biofabrication: a 21 st century manufacturing paradigm. Biofabrication 1, 022001, 2009.

13. Mironov, V., Printing technology to produce living tissue. Expert Opin Biol Ther 3, 701, 2003.

14. Mironov, V., Markwald, R.R., and Forgacs, G. Organ printing: self-assembling cell aggregates as "Bioink." Sci Med 9, 69, 2003.
15. Williams, D., Thayer, P., and Martinez, H. A perspective on the physical, mechanical and biological specifications of bioinks and the development of functional tissues in 3D bioprinting. Bioprinting 9, 19, 2018.

16. Vijayavenkataraman, S., Yan, W.C., and Lu, W.F. 3D bioprinting of tissues and organs for regenerative medicine. Adv Drug Deliv Rev 132, 296, 2018.

17. Tomasina, C., Bodet, T., and Mota, C. Bioprinting vasculature: materials, cells and emergent techniques. Materials (Basel) 12, 2701, 2019.

18. Jungst, T., Smolan, W., and Schacht, K. Strategies and molecular design criteria for 3D printable hydrogels. Chem Rev 116, 1496, 2016.

19. Ligon, S.C., Liska, R., and Stampfl, J. Polymers for 3D printing and customized additive manufacturing. Chem Rev 117, 10212, 2017.

20. Gungor-Ozkerim, P.S., Inci, I., and Zhang, Y.S. Bioinks for 3D bioprinting: an overview. Biomater Sci 6, 915, 2018.

21. Bishop, E.S., Mostafa, S., and Pakvasa, M. 3-D bioprinting technologies in tissue engineering and regenerative medicine: current and future trends. Genes Dis 4, 185, 2017.

22. Ashammakhi, N., Ahadian, S., and Xu, C. Bioinks and bioprinting technologies to make heterogeneous and biomimetic tissue constructs. Materials Today Bio 1, 100008, 2019.

23. Attalla, R., Puersten, E., and Jain, N. 3D bioprinting of heterogeneous bi- and tri-layered hollow channels within gel scaffolds using scalable multi-axial microfluidic extrusion nozzle. Biofabrication 11, 015012, 2018.

24. Štumberger, G., and Vihar, B. Freeform perfusable microfluidics embedded in hydrogel matrices. Materials 11, 2529, 2018.

25. Ji, S., Almeida, E., and Guvendiren, M. 3D bioprinting of complex channels within cell-laden hydrogels. Acta Biomater 95, 214, 2019.

26. Zhang, Y., Yu, Y., and Akkouch, A. In vitro study of directly bioprinted perfusable vasculature conduits. Biomater Sci 3, 134, 2015.

27. Schöneberg, J., De Lorenzi, F., and Theek, B. Engineering biofunctional in vitro vessel models using a multilayer bioprinting technique. Sci Rep 8, 10430, 2018.

28. Boni, R., Ali, A., and Shavandi, A. Current and novel polymeric biomaterials for neural tissue engineering. $\mathrm{J}$ Biomed Sci 25, 90, 2018.

29. Yildirimer, L., Zhang, Q., and Kuang, S. Engineering three-dimensional microenvironments towards in vitro disease models of the central nervous system. Biofabrication 11, 032003, 2019.

30. Joung, D., Truong, V., and Neitzke, C.C. 3D printed stem-cell derived neural progenitors generate spinal cord scaffolds. Adv Funct Mater 28, 1801850, 2018.

31. Ning, L., Sun, H., and Lelong, T. 3D bioprinting of scaffolds with living Schwann cells for potential nerve tissue engineering applications. Biofabrication 10, 035014, 2018.

32. Miguel, S.P., Cabral, C.S.D., and Moreira, A.F. Production and characterization of a novel asymmetric 3D printed construct aimed for skin tissue regeneration. Colloids Surf B Biointerfaces 181, 994, 2019.

33. Liu, P., Shen, H., and Zhi, Y. 3D bioprinting and in vitro study of bilayered membranous construct with human cells-laden alginate/gelatin composite hydrogels. Colloids Surf B Biointerfaces 181, 1026, 2019. 
34. Cheng, L., Yao, B., and Hu, T. Properties of an alginategelatin-based bioink and its potential impact on cell migration, proliferation, and differentiation. Int $\mathrm{J}$ Biol Macromol 135, 1107, 2019.

35. Derr, K., Zou, J., and Luo, K. Fully three-dimensional bioprinted skin equivalent constructs with validated morphology and barrier function. Tissue Eng Part C Methods 25, 334, 2019.

36. Seol, Y.J., Lee, H., and Copus, J.S. 3D bioprinted BioMask for facial skin reconstruction. Bioprinting 10, e00028, 2018.

37. Albanna, M., Binder, K.W., and Murphy, S.V. In situ bioprinting of autologous skin cells accelerates wound healing of extensive excisional full-thickness wounds. Sci Rep 9, 1856, 2019.

38. Apelgren, P., Amoroso, M., and Saljo, K. Skin grafting on $3 \mathrm{D}$ bioprinted cartilage constructs in vivo. Plast Reconstr Surg Glob Open 6, e1930, 2018.

39. Mazzocchi, A., Devarasetty, M., and Huntwork, R. Optimization of collagen type I-hyaluronan hybrid bioink for 3D bioprinted liver microenvironments. Biofabrication 11, 015003, 2018.

40. Christoffersson, J., Aronsson, C., and Jury, M. Fabrication of modular hyaluronan-PEG hydrogels to support 3D cultures of hepatocytes in a perfused liver-on-a-chip device. Biofabrication 11, 015013, 2018.

41. Lind, J.U., Busbee, T.A., and Valentine, A.D. Instrumented cardiac microphysiological devices via multimaterial three-dimensional printing. Nat Mater 16, 303, 2017.

42. Wang, Z., Lee, S.J., and Cheng, H.J. 3D bioprinted functional and contractile cardiac tissue constructs. Acta Biomater 70, 48, 2018.

43. Noor, N., Shapira, A., and Edri, R. 3D printing of personalized thick and perfusable cardiac patches and hearts. Adv Sci 6, 1900344, 2019.

44. Shapira, A., Noor, N., and Asulin, M. Stabilization strategies in extrusion-based 3D bioprinting for tissue engineering. Appl Phys Rev 5, 041112, 2018.

45. Lee, A., Hudson, A.R., and Shiwarski, D.J. 3D bioprinting of collagen to rebuild components of the human heart. Science 365, 482, 2019.

46. Sticker, D., Rothbauer, M., and Ehgartner, J. Oxygen management at the microscale: a functional biochip material with long-lasting and tunable oxygen scavenging properties for cell culture applications. ACS Appl Mater Interfaces 11, 9730, 2019.

47. Arai, K., Murata, D., and Verissimo, A.R. Fabrication of scaffold-free tubular cardiac constructs using a Bio-3D printer. PLoS One 13, e0209162, 2018.

48. Skylar-Scott, M.A., Uzel, S.G.M., and Nam, L.L. Biomanufacturing of organ-specific tissues with high cellular density and embedded vascular channels. Sci Adv 5, eaaw2459, 2019.

49. Pitsalidis, C., Ferro, M.P., and Iandolo, D. Transistor in a tube: a route to three-dimensional bioelectronics. Sci Adv 4, eaat4253, 2018.

50. Ali, M., and Pr, A.K. A photo-crosslinkable kidney ECMderived bioink accelerates renal tissue formation. Adv Healthc Mater 8, e1800992, 2019.

51. Kim, W., Kim, M., and Kim, G.H. 3D-printed biomimetic scaffold simulating microfibril muscle structure. Adv Funct Mater 28, 1800405, 2018.
52. Kim, W., Lee, H., and Lee, J. Efficient myotube formation in 3D bioprinted tissue construct by biochemical and topographical cues. Biomaterials 230, 119632, 2019.

53. Lee, H., Kim, W., and Lee, J. Effect of hierarchical scaffold consisting of aligned dECM nanofibers and poly(lactide-co-glycolide) struts on the orientation and maturation of human muscle progenitor cells. ACS Appl Mater Interfaces 11, 39449, 2019.

54. Kim, J.H., Seol, Y-J., and Ko, I.K. 3D bioprinted human skeletal muscle constructs for muscle function restoration. Sci Rep 8, 12307, 2018.

55. Li, L., Li, J., and Guo, J. 3D molecularly functionalized cell-free biomimetic scaffolds for osteochondral regeneration. Adv Funct Mater 29, 1807356, 2019.

56. Taniguchi, D., Matsumoto, K., and Tsuchiya, T. Scaffoldfree trachea regeneration by tissue engineering with bio3D printing. Interact Cardiovasc Thorac Surg 26, 745, 2018.

57. Mekhileri, N.V., Lim, K.S., and Brown, G.C.J. Automated 3D bioassembly of micro-tissues for biofabrication of hybrid tissue engineered constructs. Biofabrication 10, 024103, 2018.

58. Castilho, M., Mouser, V., and Chen, M. Bi-layered microfibre reinforced hydrogels for articular cartilage regeneration. Acta Biomater 95, 297, 2019.

59. Lai, Y., Li, Y., and Cao, H. Osteogenic magnesium incorporated into PLGA/TCP porous scaffold by 3D printing for repairing challenging bone defect. Biomaterials 197, 207, 2019.

60. Choe, G., Oh, S., and Seok, J.M. Graphene oxide/alginate composites as novel bioinks for three-dimensional mesenchymal stem cell printing and bone regeneration applications. Nanoscale 11, 23275, 2019.

61. Wei, L., Wu, S., and Kuss, M. 3D printing of silk fibroin-based hybrid scaffold treated with platelet rich plasma for bone tissue engineering. Bioact Mater 4, 256, 2019.

62. Miri, A.K., Mostafavi, E., and Khorsandi, D. Bioprinters for organs-on-chips. Biofabrication 11, 042002, 2019.

63. Yi, H.G., Lee, H., and Cho, D.W. 3D printing of organson-chips. Bioengineering (Basel) 4, E10, 2017.

64. Miri, A.K., Nieto, D., and Iglesias, L. Microfluidicsenabled multimaterial maskless stereolithographic bioprinting. Adv Mater 30, e1800242, 2018.

65. Wang, Z., Abdulla, R., and Parker, B. A simple and highresolution stereolithography-based 3D bioprinting system using visible light crosslinkable bioinks. Biofabrication 7, 045009, 2015.

66. Murphy, S.V., and Atala, A. 3D bioprinting of tissues and organs. Nat Biotechnol 32, 773, 2014.

67. Chartrain, N.A., Williams, C.B., and Whittington, A.R. A review on fabricating tissue scaffolds using vat photopolymerization. Acta Biomater 74, 90, 2018.

68. Grigoryan, B., and Paulsen, S.J. Multivascular networks and functional intravascular topologies within biocompatible hydrogels. Science 364, 458, 2019.

69. Grix, T., Ruppelt, A., and Thomas, A. Bioprinting perfusion-enabled liver equivalents for advanced organon-a-chip applications. Genes (Basel) 9, E176, 2018.

70. Parrish, J., Lim, K.S., and Baer, K. A 96-well microplate bioreactor platform supporting individual dual perfusion and high-throughput assessment of simple or biofabricated 3D tissue models. Lab Chip 18, 2757, 2018. 
71. Huebsch, N., Charrez, B., and Siemons, B. Metabolicallydriven maturation of hiPSC-cell derived heart-on-a-chip. bioRxiv 485169, 2018.

72. Caluori, G., Pribyl, J., and Pesl, M. Non-invasive electromechanical cell-based biosensors for improved investigation of 3D cardiac models. Biosens Bioelectron 124, $129,2019$.

73. Shima, A., Morimoto, Y., and Sweeney, H.L. Threedimensional contractile muscle tissue consisting of human skeletal myocyte cell line. Exp Cell Res 370, 168, 2018.

74. Bernal, P.N., Delrot, P., and Loterie, D. Volumetric bioprinting of complex living-tissue constructs within seconds. Adv Mater 31, e1904209, 2019.

75. Antoshin, A.A., Churbanov, S.N., and Minaev, N.V. LIFT-bioprinting, is it worth it? Bioprinting 15, e00052, 2019.

76. Guillotin, B., Ali, M., and Ducom, A. Chapter 6-LaserAssisted Bioprinting for Tissue Engineering. In: Forgacs, G., and Sun, W., eds. Biofabrication. Boston: William Andrew Publishing, 2013, pp. 95-118.

77. Kérourédan, O., Bourget, J.M., and Remy M. Micropatterning of endothelial cells to create a capillary-like network with defined architecture by laser-assisted bioprinting. J Mater Sci Mater Med 30, 28, 2019.

78. Kérourédan, O., Hakobyan, D., and Remy, M. In situ prevascularization designed by laser-assisted bioprinting: effect on bone regeneration. Biofabrication 11, 045002, 2019.

79. Sorkio, A., Koch, L., and Koivusalo, L. Human stem cell based corneal tissue mimicking structures using laserassisted 3D bioprinting and functional bioinks. Biomaterials 171, 57, 2018.

80. Koch, L., Deiwick, A., and Franke, A. Laser bioprinting of human induced pluripotent stem cells-the effect of printing and biomaterials on cell survival, pluripotency, and differentiation. Biofabrication 10, 035005, 2018.

81. Kim, S.H., Yeon, Y.K., and Lee, J.M. Precisely printable and biocompatible silk fibroin bioink for digital light processing 3D printing. Nat Commun 9, 1620, 2018.

82. Dobos, A., Van Hoorick, J., and Steiger, W. Thiolgelatin-norbornene bioink for laser-based high-definition bioprinting. Adv Healthc Mater e1900752, 2019.

83. Hauptmann, N., Lian, Q., and Ludolph, J. Biomimetic designer scaffolds made of D,L-lactide-varepsiloncaprolactone polymers by 2-photon polymerization. Tissue Eng Part B Rev 25, 167, 2019.

84. Pennacchio, F.A., Casale, C., and Urciuolo, F. Controlling the orientation of a cell-synthesized extracellular matrix by using engineered gelatin-based building blocks. Biomater Sci 6, 2084, 2018.

85. Holzer, B., Lunzer, M., and Rosspeintner, A. Towards efficient initiators for two-photon induced polymerization: fine tuning of the donor/acceptor properties. Mol Syst Design Eng 4, 437, 2019.

86. Van Hoorick, J., Gruber, P., and Markovic, M. Highly reactive thiol-norbornene photo-click hydrogels: toward improved processability. Macromol Rapid Commun 39, e1800181, 2018.

87. Heintz, K.A., Bregenzer, M.E., and Mantle, J.L. Fabrication of 3D biomimetic microfluidic networks in hydrogels. Adv Healthc Mater 5, 2153, 2016.

88. Guo, J., Keller, K.A., and Govyadinov, P. Accurate flow in augmented networks (AFAN): an approach to gen- erating three-dimensional biomimetic microfluidic networks with controlled flow. Anal Methods 11, 8, 2019.

89. Kirillova, A., Maxson, R., and Stoychev, G. 4D biofabrication using shape-morphing hydrogels. Adv Mater 29, $1900752,2017$.

90. Miri, A.K., Khalilpour, A., and Cecen, B. Multiscale bioprinting of vascularized models. Biomaterials 198, 204, 2019.

91. Gao, B., Yang, Q., and Zhao, X. 4D bioprinting for biomedical applications. Trends Biotechnol 34, 746, 2016.

92. Ionov, L. 4D biofabrication: materials, methods, and applications. Adv Healthc Mater 7, e1800412, 2018.

93. Serpooshan, V., Hu, J., and Chirikian, O. 3D Printing Applications in Cardiovascular Medicine. Amsterdam, The Netherlands: Elsevier, 2018.

94. Stoychev, G., Puretskiy, N., and Ionov, L. Self-folding allpolymer thermoresponsive microcapsules. Soft Matter 7, 3277, 2011.

95. Deng, H., Dong, Y., and Su, J-W. Bioinspired programmable polymer gel controlled by swellable guest medium. ACS Appl Mater Interfaces 9, 30900, 2017.

96. Su, J.-W., Tao, X., and Deng, H. 4D printing of a selfmorphing polymer driven by a swellable guest medium. Soft Matter 14, 765, 2018.

97. Zhang, L., Zuo, X., and Li, S. Synergistic therapy of magnetism-responsive hydrogel for soft tissue injuries. Bioact Mater 4, 160, 2019.

98. Betsch, M., Cristian, C., and Lin, Y.Y. Incorporating 4D into bioprinting: real-time magnetically directed collagen fiber alignment for generating complex multilayered tissues. Adv Healthc Mater 7, 1800894, 2018.

99. Miao, S., Cui, H., and Nowicki, M. Photolithographicstereolithographic-tandem fabrication of 4D smart scaffolds for improved stem cell cardiomyogenic differentiation. Biofabrication 10, 035007, 2018.

100. Miao, S., Nowicki, M., and Cui, H. 4D anisotropic skeletal muscle tissue constructs fabricated by staircase effect strategy. Biofabrication 11, 035030, 2019.

101. Miao, S., Cui, H., and Nowicki, M. Stereolithographic 4D bioprinting of multiresponsive architectures for neural engineering. Adv Biosyst 2, 1800101, 2018.

102. Parameswaran-Thankam, A., Parnell, C.M, and Watanabe, F. Guar-based injectable thermoresponsive hydrogel as a scaffold for bone cell growth and controlled drug delivery. ACS Omega 3, 15158, 2018.

103. Zhao, C., Zeng, Z., and Qazvini, N.T. Thermoresponsive citrate-based graphene oxide scaffold enhances bone regeneration from BMP9-stimulated adipose-derived mesenchymal stem cells. ACS Biomater Sci Eng 4, 2943, 2018.

104. Vojtova, L., Michlovska, L., and Valova, K. The effect of the thermosensitive biodegradable PLGA-PEG-PLGA copolymer on the rheological, structural and mechanical properties of thixotropic self-hardening tricalcium phosphate cement. Int J Mol Sci 20, 391, 2019.

105. Guduric, V., Siadous, R., and Babilotte, J. Layer-by-layer bioassembly of poly(lactic) acid membranes loaded with coculture of HBMSCs and EPCs improves vascularization in vivo. J Biomed Mater Res A 107, 2629, 2019.

106. Li, J., and Wang, H. Magnetic micromachine using nickel nanoparticles for propelling and releasing in indirect assembly of cell-laden micromodules. Micromachines (Basel) 10, E370, 2019. 
107. Mi, S., Yi, X., and Du, Z. Construction of a liver sinusoid based on the laminar flow on chip and self-assembly of endothelial cells. Biofabrication 10, 025010, 2018.

108. Flegeau, K., Rubin, S., and Mucha, S. Towards an in vitro model of glomerular barrier unit with an innovative bioassembly method. Nephrol Dial Transplant 2019 [Epub ahead of print]; DOI: 10.1093/ndt/gfz094.

109. Kannan, S., The 3D bioprinting revolution. Harv Sci Rev 2014.

110. Derakhshanfar, S., Mbeleck, R., and Xu, K. 3D bioprinting for biomedical devices and tissue engineering: a review of recent trends and advances. Bioact Mater 3, 144, 2018.

111. Ke, D., and Murphy, S.V. Current challenges of bioprinted tissues toward clinical translation. Tissue Eng Part B Rev 25, 1, 2019.

112. Robert, J., and Button, E.B. Clearance of beta-amyloid is facilitated by apolipoprotein $\mathrm{E}$ and circulating high- density lipoproteins in bioengineered human vessels. Elife 6, e29595, 2017.

Address correspondence to: Lorenzo Moroni, PhD Complex Tissue Regeneration Department MERLN Institute for Technology-Inspired Regenerative Medicine Maastricht University Universiteitssingel 40 Maastricht 6229ER The Netherlands

E-mail:1.moroni@maastrichtuniversity.nl

Received: December 18, 2019

Accepted: December 18, 2019

Online Publication Date: January 21, 2020 